\title{
AN ANALYSIS OF PULSAR NULLING STATISTICS
}

\author{
JAMES D. BIgGS \\ Nuffield Radio Astronomy Laboratories, University of Manchester
}

\begin{abstract}
We have sought correlations between the fraction of null pulses with other pulsar parameters for an ensemble of 72 pulsars using survival analysis methods. The strongest correlation was found between the null fraction and pulse period. Correlations were also found between other parameters that typically have strong dependencies on pulse period, and this tends to indicate that the null fraction increases with age as was first suggested by Ritchings (1976). However, no explicit correlation was found between pulsar characteristic age and null fraction. A significant anti-correlation was found between the angle subtended by the magnetic and rotation axes and the null fraction.

Many of the pulsars presented here were found to null. In particular, all pulse profile classes in the scheme devised by Rankin (1983a) have members that null. Differences in the mean age of these pulsar classes are not very pronounced, and the influence of class on pulse nulling statistics is probably less than that suggested by Rankin (1986), but cannot entirely be ruled out. Also, there is considerable variation in the fraction of null pulses from pulsars within each class, but generally class $S_{\mathfrak{t}}$ pulsars null the least. Of special note is the fact that two pulsars PSR 0833-45 and PSR 1556-44 apparently do not null. The upper limit for PSR 0833-45 is quite low; no nulls were detected in observations of over 120,000 pulses.

The similarity of the nulling parameters of pulsars observed at two frequencies near $400 \mathrm{MHz}$ and $843 \mathrm{MHz}$ suggests that the pulsar emission mechanism is wide band over this frequency range.
\end{abstract}

\section{Introduction}

Pulsed radio emission from pulsars is variable on many time scales and sometimes appears to cease (or is greatly diminished). Typically, the time scale for these null pulses is 1 to a few pulse periods; however, it may be many hours in duration as is the case for PSR 0826-34 (Durdin et al. 1979). The nulling phenomenon is a vital clue to understanding the elusive pulsar emission mechanism, since it profoundly affects the subpulses that are thought to be a basic unit of emission. More specifically, nulling seems to have a widespread influence over the pulsar polar cap since core (those nearly aligned with the magnetic axis) and cone (those near the edge of the emission cone) components null simultaneously (Rankin 1986). Also, detailed observations by Lyne and Ashworth (1983) have shown that the mechanism responsible for regular drifting subpulses across the pulse window of PSR 0809+74 and PSR 0818-13 apparently continues at a reduced rate through a null and exponentially relaxes back to the original drift rate after a null, with a time scale proportional to the null duration. For PSR 0809+74 Smirnova (1983) has shown that an increase in subpulse drift rate is accompanied by an increase in subpulse flux density. However, a detailed study of the drifting subpulses from PSR 1944+17 (Deich et al. 1986) did not find any of the phenomena outlined above associated with its nulls.
An early investigation of pulse nulling in an ensemble of pulsars by Ritchings (1976) suggested that old pulsars have a higher fraction of null pulses than younger pulsars. This implied that old pulsars cease to be observable because they spend an increasing fraction of time in a null state.

A more recent analysis of pulse nulling statistics by Rankin (1986) found that the fraction of null pulses $(N F)$ does not increase with pulsar age alone. $N F$ also depends on the class $\left[S_{\mathfrak{t}}, T, M\right.$, $S_{d}$ or $D$ in the nomenclature of Rankin (1983a)] of pulse profile. In particular, Rankin found that class $S_{\mathfrak{t}}$ pulsars have rather low values of $N F$ (and may not null at all) and are on average younger than other classes of pulsars, and thus the correlation found by Ritchings (1976) also depends on the pulsar class. Also, within a particular pulsar class no increase in $N F$ with age was found. However, within most of the pulsar classes, the limits on for the various pulsars varied widely, and in some classes by about 3 orders of magnitude.

A substantial fraction of the pulsar nulling statistics are upper limits and standard statistical methods are unsuited to determine such things as correlation coefficients from this type of data. However, over the last few years a class of statistics called survival analysis (see Isobe et al. 1986, and the references therein) has been developed to analyze censored data (upper or lower limits). Generally, we used the methods described by Isobe et al., namely, Cox's proportion hazard model and the 
Table 1 Parameters of pulsars with known nulling statistics (continued)

\begin{tabular}{|c|c|c|c|c|c|c|c|c|c|c|c|c|c|}
\hline PSR & $\begin{array}{c}P \\
(\mathrm{~s})\end{array}$ & $\begin{array}{c}\dot{P} \times 10^{15} \\
\left(\mathrm{ss}^{-1}\right)\end{array}$ & $\begin{array}{l}\log \tau \\
(y r)\end{array}$ & $\begin{array}{c}\log \left(B_{\mathbf{g}} \sin \alpha\right) \\
(\mathrm{G})\end{array}$ & $\begin{array}{c}\log \mathcal{L} \\
\left(\mathrm{mJykpc}^{2}\right)\end{array}$ & $\begin{array}{c}\log \dot{E} \\
\left(\operatorname{ergs}^{-1}\right)\end{array}$ & $\begin{array}{c}\log B_{L C} \\
(G)\end{array}$ & $Q$ & $\underset{\left({ }^{\circ}\right)}{\alpha_{\mathrm{LM}}}$ & $\begin{array}{l}\alpha_{R} \\
\left({ }^{\circ}\right)\end{array}$ & $\begin{array}{l}\text { Nulls } \\
(\%)\end{array}$ & p. & Ref \\
\hline \multicolumn{14}{|c|}{ Multiple component pulsars (M) } \\
\hline $\begin{array}{l}0523+11 \\
0826-34\end{array}$ & $\begin{array}{l}0.354 \\
1.849\end{array}$ & $\begin{array}{l}0.07 \\
1.00\end{array}$ & $\begin{array}{l}7.90 \\
7.47\end{array}$ & $\begin{array}{l}11.2 \\
12.1\end{array}$ & $\begin{array}{l}2.31 \\
0.51\end{array}$ & $\begin{array}{l}31.8 \\
30.8\end{array}$ & $\begin{array}{c}1.11 \\
-1.34\end{array}$ & $\begin{array}{l}1.84 \\
3.93\end{array}$ & $\begin{array}{l}49.1 \\
10.0\end{array}$ & $\begin{array}{c}78.0 \\
3.0\end{array}$ & $\begin{array}{l}\leq 0.06 \\
\bar{Y} ; 70 \pm 35^{*} \\
Y ; \leq 12\end{array}$ & $\begin{array}{l}\overline{1} .0 \pm 0.2\end{array}$ & $\begin{array}{l}4 \\
3 \\
9\end{array}$ \\
\hline $\begin{array}{l}0940+16 \\
1237+25 \\
1737+13\end{array}$ & $\begin{array}{l}1.087 \\
1.382 \\
0.803\end{array}$ & $\begin{array}{l}0.90 \\
0.96 \\
1.45\end{array}$ & $\begin{array}{l}7.28 \\
7.36 \\
6.94\end{array}$ & $\begin{array}{l}12.0 \\
12.1 \\
12.0\end{array}$ & $\begin{array}{l}1.00 \\
1.24 \\
2.36\end{array}$ & $\begin{array}{l}31.4 \\
31.2 \\
32.0\end{array}$ & $\begin{array}{c}-0.23 \\
-0.72 \\
0.64\end{array}$ & $\begin{array}{l}2.29 \\
2.90 \\
1.35\end{array}$ & $\begin{array}{r}-\overline{-} \\
40.8\end{array}$ & $\begin{array}{l}5 \overline{1.0} \\
42.0\end{array}$ & $\begin{array}{l}Y ; 8 \pm 3 \\
Y ; 6.0 \pm 2.5 \\
\leq 0.02\end{array}$ & $\overline{0} .75 \pm 0.2$ & $\begin{array}{l}4 \\
2 \\
4\end{array}$ \\
\hline $\begin{array}{l}1857-26 \\
1919+21\end{array}$ & $\begin{array}{l}0.612 \\
1.337\end{array}$ & $\begin{array}{l}0.16 \\
1.35\end{array}$ & $\begin{array}{l}7.78 \\
7.20\end{array}$ & $\begin{array}{l}11.5 \\
12.1\end{array}$ & $\begin{array}{l}2.31 \\
1.42\end{array}$ & $\begin{array}{l}31.4 \\
31.3\end{array}$ & $\begin{array}{c}0.27 \\
-0.50\end{array}$ & $\begin{array}{l}2.43 \\
2.44\end{array}$ & $\begin{array}{l}20.9 \\
45.4\end{array}$ & $\begin{array}{l}23.0 \\
45.0\end{array}$ & $\begin{array}{l}Y ; 10.0 \pm 2.5 \\
\leq 0.25\end{array}$ & $\begin{array}{l}1.1 \pm 0.2 \\
0.95 \pm 0.1\end{array}$ & $\begin{array}{l}2 \\
2\end{array}$ \\
\hline \multicolumn{14}{|c|}{ Conal single pulsars $\left(S_{d}\right)$} \\
\hline $0031-07$ & 0.943 & 0.41 & 7.56 & 11.8 & 0.58 & 31.3 & -0.26 & 2.68 & - & - & $\begin{array}{l}Y ; 37.7 \pm 0.1^{*} \\
Y<<16\end{array}$ & $\overrightarrow{1} 2+0$ & 1 \\
\hline $0628-28$ & 1.244 & 7.11 & 6.44 & 12.5 & 2.18 & 32.2 & 0.38 & 1.16 & 15.8 & - & $\leq 2$ & $\begin{array}{l}0.85 \pm 0.1 \\
0.8 \pm 0.2\end{array}$ & $\begin{array}{l}2 \\
9\end{array}$ \\
\hline $0809+74$ & 1.292 & 0.17 & 8.09 & 11.7 & 0.16 & 30.5 & -1.33 & 5.41 & & - & $Y ; 1.42 \pm 0.02$ & $0.9 \pm 0.2$ & 2,5 \\
\hline $0818-13$ & 1.238 & 2.11 & 6.97 & 12.2 & 2.31 & 31.6 & -0.14 & 1.88 & 90.0 & - & $\begin{array}{l}Y ; 1.01 \pm 0.01^{*} \\
Y ; 1 \pm 0.5\end{array}$ & $\begin{array}{l}0.9 \pm 0.2^{*} \\
0.8 \pm 0.1\end{array}$ & $\begin{array}{c}2,5 \\
9\end{array}$ \\
\hline $0820+02$ & 0.865 & 0.10 & 8.12 & 11.5 & 1.19 & 30.8 & -0.67 & 4.22 & - & - & $\leq 0.06$ & - & 4 \\
\hline $0950+08$ & 0.253 & 0.23 & 7.24 & 11.4 & 1.11 & 32.7 & 2.34 & 0.80 & 10.0 & - & & $0.2 \pm 0.1$ & 2 \\
\hline $1612+07$ & 1.207 & 2.36 & 6.91 & 12.2 & 0.85 & 31.7 & -0.04 & 1.75 & - & - & & - & 4 \\
\hline $1923+04$ & 1.074 & 2.46 & 6.84 & 12.2 & 2.06 & 31.9 & 0.24 & 1.51 & - & - & & & 4 \\
\hline $2016+28$ & 0.558 & 0.15 & 7.77 & 11.5 & 2.40 & 31.5 & 0.44 & 2.26 & 40.4 & - & $\leq 0.25$ & $0.95 \pm 0.1$ & 2 \\
\hline $2021+51$ & 0.529 & 3.05 & 6.44 & 12.1 & 1.44 & 32.9 & 1.87 & 0.63 & $3-$ & - & $\overline{\leq} 5$ & $0.4 \pm 0.2$ & 2 \\
\hline $2303+30$ & 1.576 & 2.90 & 6.94 & 12.3 & 1.96 & 31.5 & -0.53 & 2.16 & - & - & $1.0 \pm 0.5$ & - & 7 \\
\hline $2315+21$ & 1.445 & 1.05 & 7.34 & 12.1 & 0.83 & 31.1 & -0.78 & 2.94 & - & - & $Y ; 3.0 \pm 0.5$ & - & 4 \\
\hline \multicolumn{14}{|c|}{ Conal double pulsars (D) } \\
\hline $0148-06$ & 1.465 & 0.44 & 7.72 & 11.9 & 1.52 & 30.7 & -1.18 & 4.21 & 15.5 & - & $Y ; \leq 5$ & $0.85 \pm 0.1$ & 9 \\
\hline $0301+19$ & 1.388 & 1.30 & 7.23 & 12.1 & 0.94 & 31.3 & -0.60 & 2.59 & 31.9 & - & $Y ; \overline{10} \pm 5$ & & 7 \\
\hline $0525+21$ & 3.745 & 40.1 & 6.17 & 13.1 & 2.57 & 31.5 & -1.26 & 1.95 & 23.2 & - & $Y: 25 \pm 5$ & $0.7 \pm 0.2$ & 2 \\
\hline $0751+32$ & 1.442 & 1.07 & 7.33 & 12.1 & 1.53 & 31.1 & -0.76 & 2.91 & - & - & $Y ; 34.0 \pm 0.5$ & & 4 \\
\hline $6834+06$ & 1.274 & 6.80 & 6.47 & 12.5 & 1.08 & 32.1 & 0.31 & 1.21 & 60.7 & - & $Y ; 7.1 \pm 0.1$ & $0.85 \pm 0.1$ & 2 \\
\hline $1133+16$ & 1.188 & 3.73 & 6.70 & 12.3 & 0.88 & 31.9 & 0.20 & 1.43 & 51.3 & - & $Y ; 15.0 \pm 2.5$ & $0.3 \pm 0.1$ & 2 \\
\hline $1530+27$ & 1.125 & 0.80 & 7.35 & 12.0 & 0.45 & 31.3 & -0.35 & 2.48 & $3-$ & - & $\mathrm{Y} ; 6 \pm 2$ & - & 4 \\
\hline $1942-00$ & 1.046 & 0.54 & 7.49 & 11.9 & 2.20 & 31.3 & -0.37 & 2.70 & 29.2 & - & $Y ; 21 \pm 1$ & - & 4 \\
\hline $1944+17$ & 0.441 & 0.02 & 8.46 & 11.0 & 1.04 & 31.0 & 0.16 & 3.61 & $1-$ & - & $Y ; 64 \pm 32$ & $0.45 \pm 0.2$ & 2,6 \\
\hline $2044+15$ & 1.138 & 0.19 & 7.99 & 11.7 & 1.37 & 30.7 & -1.01 & 4.53 & 40.7 & - & $\leq 0.04$ & - & 4 \\
\hline $2154+40$ & 1.525 & 3.42 & 6.85 & 12.4 & 2.42 & 31.6 & -0.38 & 1.95 & 522.4 & $=$ & $Y ; 7.5 \pm 2.5$ & $0.75 \pm 0.2$ & 2 \\
\hline
\end{tabular}

- Value used ir the analysis.

$Y$ Indicates that null pulses have been observed.

References.--(1) Huguenin, Taylor and Troland 1970. (2) Ritchings 1976. (3) Durdin et al. 1979. (4) Backus 1981. (5) Lyne and Ashworth 1983. (6) Deich et al. 1986. (7) Rankin 1986. (8) Nowakowski and Hankins 1986 (reported in Rankin 1986). (9) Biggs 1986; and this paper.

pulses for PSR 1556-44 (class T) at $645 \mathrm{MHz}$.

No correlations significant at $<0.02$ were found between $N F$ and the pulsar parameters for class $\mathbf{S}_{\mathrm{d}}$, $D, S_{t}$ and $M$ pulsars. This is probably the result of the small numbers of pulsars in these classes. When the $\mathbf{S}_{d}$ and $\mathbf{D}$ pulsars were combined into one group, again no significant correlations were found. Correlations significant at $<0.02$ were, however, found for the groups composed of pulsars with (i) $\mathbf{T}$ class profiles, (ii) profiles with core components, and (iii) any profile class (i.e., all the puls.ırs). These are given in table 2 as well as the region of parameter space that is incompatible with the correlation. Generally the most significant correlations were found with $P$, $B_{\mathrm{LC}}, Q, \alpha_{\mathrm{LM}}$ and $\alpha_{\mathrm{R}}$. None of the correlations were found to be significantly influenced by outlying data (such as those of the Vela pulsar). The relationships between $N F, P$ and $\log \mathcal{L}$ for all the data are plotted in figure 1. Also plotted are the relationships between $N F, \alpha_{\mathrm{LM}}$ and $\alpha_{\mathrm{R}}$ for the pulsars with core emission.

Table 2 indicates that $P$ has the most signifi- 

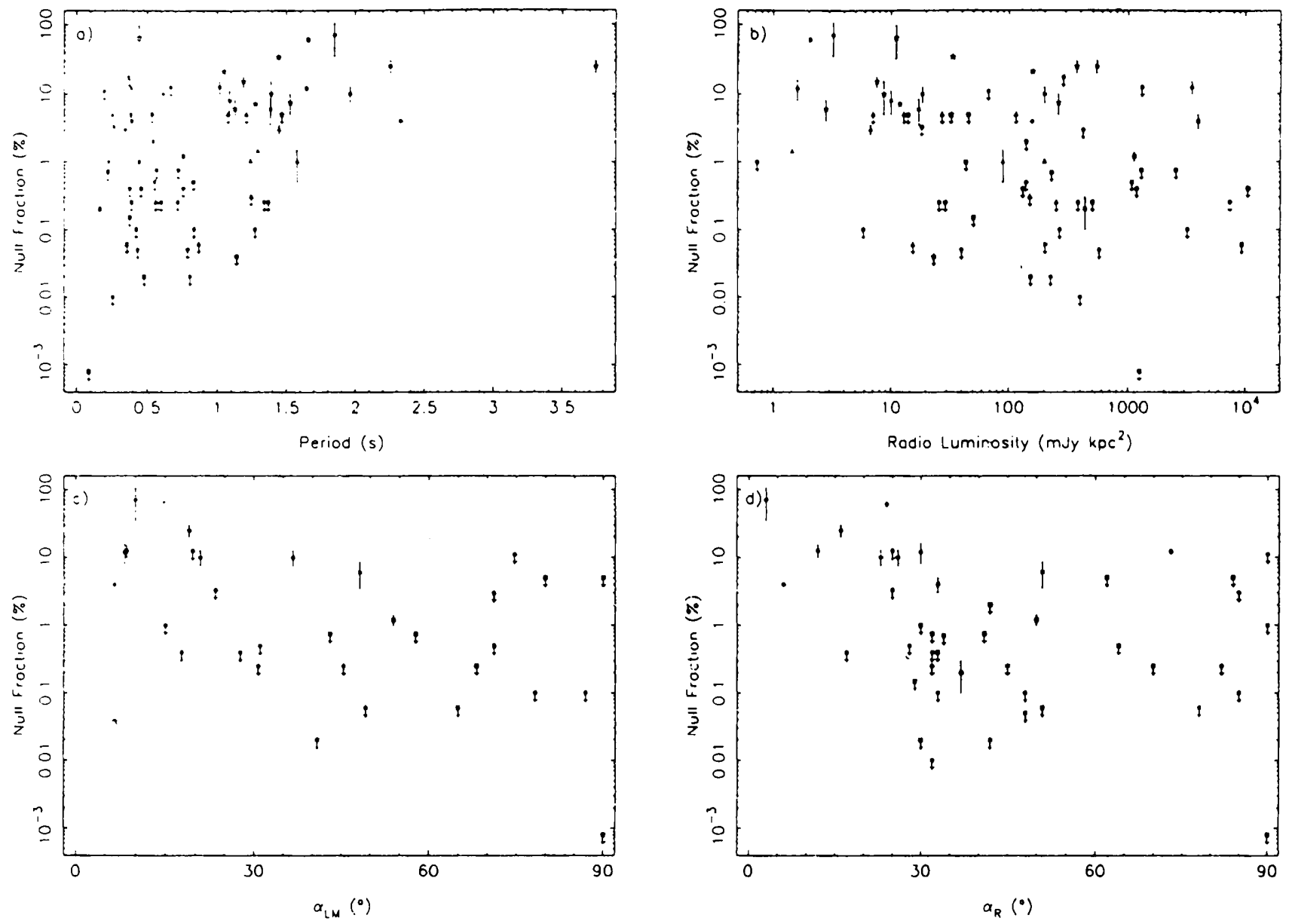

Figure 1 Fraction of null pulses as a function of $(a)$ pulse period, $(b)$ radio luminosity, $(c)$ and $(d)$ the angle between the magnetic and rotation axes $(\alpha)$ calculated by Lyne and Manchester (1988) and Rankin (1990), respectively, for class $S_{t}$ $(\boldsymbol{\square}), \mathbf{T}(\bullet), \mathbf{M}(*), \mathbf{S}_{\mathbf{d}}(\Delta)$ and $\mathbf{D}(\star)$ pulsars.

cant and persistent correlation with $N F$. The correlation implies that pulsars with small $P$ do not null frequently. Conversely, large values of $N F$ are commonly found in long period pulsars, and this may indicate that the pulse emission mechanism is faltering for these objects since quantities related to polar cap emission mechanisms such as the electric field parallel to the magnetic field and the maximum potential difference across the magnetosphere gap are inversely related to $P$ (Manchester and Taylor 1977 , and the references therein). However, as no significant correlation was found between $\log \tau$ and $N F$ it appears that age is not the predominant factor even though $\tau$ has a strong dependence on $P$. It should be reiterated that the correlation does not imply a deterministic relationship between $N F$ and $P$ since $N F$ can vary by over 3 and 2 orders of magnitude for $P<0.5 \mathrm{~s}$ and $P \approx 1.0 \mathrm{~s}$ pulsars, respectively (see figure 1a).

The pulse-shape class influence on the $N F$ and $P$ correlation found is still apparent but is probably less significant than proposed by Rankin (1986). We still find a degree of age stratification between the classes since the $S_{\mathfrak{t}}$ pulsars in table 1 tend to be younger than the class $T$ stars, which in turn tend to be younger than class $\mathbf{M}$ pulsars and have $\langle\log \tau\rangle(\tau$ in years $)=6.24 \pm 0.76,6.48 \pm 0.73$ and $7.42 \pm 0.13$, respectively; but its significance is much reduced as a result of the large age dispersion. Class $S_{d}$ and $D$ pulsars have similar ages and are relatively old with $\langle\log \tau\rangle=7.22 \pm 0.57$ and $7.25 \pm 0.67$, respectively. Also, within a given class Rankin found no correlation between $N F$ and age, and the pulsars in table 1 that have been observed to null are generally about the same age or slightly younger than others in their own class though the significance of this is reduced owing to the large uncertainties in age. It should be noted that selection effects may have influenced the work of Rankin (1986) because it is easier to detect the absence of pulsed emission from pulsars with large flux density, and these tend to be young and have smaller $P$ though this effect tends to be offset by the larger sample integration times possible with long period pulsars. Also, detection of the absence of pulsed emission is sensitive to the equipment used for observation and the analysis procedure, and these factors are not uniform for the data in table 1.

Correlations found between $N F$ and $\log \dot{E}$, $\log B_{\mathrm{LC}}$ and $Q$ are probably not revealing anything 
Table 2 Significance of survival analysis correlations with null fraction

\begin{tabular}{|c|c|c|c|}
\hline $\begin{array}{l}\text { para- } \\
\text { meter }\end{array}$ & $\begin{array}{c}\text { Cox's } \\
\text { method } \\
(\%)\end{array}$ & $\begin{array}{c}\text { generalized } \\
\text { Kendall's } \tau \\
\text { (\%) }\end{array}$ & $\begin{array}{l}\text { parameter } \\
\text { space } \\
\text { avoided by } \\
\text { large (small) } \\
N F\end{array}$ \\
\hline \multicolumn{4}{|c|}{ Triple component pulsars (T) } \\
\hline $\begin{array}{l}P \\
\log B_{\mathrm{LC}} \\
Q \\
\log B_{\mathrm{LM}} \\
\alpha_{\mathrm{R}} \\
\log B_{\mathrm{R}}\end{array}$ & $\begin{array}{l}0.03 \\
0.69 \\
0.76 \\
0.80 \\
7.02 \\
1.07\end{array}$ & $\begin{array}{l}0.58 \\
1.46 \\
3.35 \\
1.34 \\
1.64 \\
1.11\end{array}$ & $\begin{array}{l}\text { (large) } P \\
\text { large } \log B_{\mathrm{LC}} \\
\text { small } Q \\
\text { small } \log B_{\mathrm{LM}} \\
\text { large } \alpha_{\mathrm{R}} \\
\text { small } \log B_{\mathrm{R}}\end{array}$ \\
\hline \multicolumn{4}{|c|}{ Pulsars with core components } \\
\hline $\begin{array}{l}P \\
\log \dot{E} \\
\log B_{\mathrm{LC}} \\
Q \\
\alpha_{\mathrm{LM}} \\
\sin \alpha_{\mathrm{LM}} \\
\log B_{\mathrm{LM}} \\
\alpha_{\mathrm{R}} \\
\sin \alpha_{\mathrm{R}} \\
\log B_{\mathrm{R}}\end{array}$ & $\begin{array}{c}<0.01 \\
1.15 \\
0.23 \\
<0.01 \\
0.25 \\
0.09 \\
0.24 \\
0.60 \\
0.71 \\
0.09\end{array}$ & $\begin{array}{l}0.03 \\
0.13 \\
0.05 \\
0.22 \\
0.20 \\
0.20 \\
1.84 \\
0.23 \\
0.86 \\
0.20\end{array}$ & $\begin{array}{l}\text { (large) } P \\
\text { large } \log E \\
\text { large } \log B_{\mathrm{LC}} \\
\text { small } Q \\
\text { large } \alpha_{\mathrm{LM}} \\
\text { large } \sin \alpha_{\mathrm{LM}} \\
\text { small } \log B_{\mathrm{LM}} \\
\text { large } \alpha_{\mathrm{R}} \\
\text { large } \sin \alpha_{\mathrm{R}} \\
\text { small } \log B_{\mathrm{R}}\end{array}$ \\
\hline \multicolumn{4}{|c|}{ All pulsars } \\
\hline $\begin{array}{l}P \\
\log \mathcal{L} \\
\log \dot{E} \\
\log B_{\mathrm{LC}} \\
Q \\
\alpha_{\mathrm{LM}} \\
\sin \alpha_{\mathrm{LM}} \\
\log B_{\mathrm{LM}}\end{array}$ & $\begin{array}{l}<0.01 \\
0.23 \\
0.06 \\
0.01 \\
0.02 \\
2.93 \\
2.03 \\
0.10\end{array}$ & $\begin{array}{c}0.01 \\
0.27 \\
<0.01 \\
<0.01 \\
<0.01 \\
0.15 \\
0.15 \\
0.45\end{array}$ & $\begin{array}{l}\text { (large) } P \\
\text { large } \log \mathcal{L} \\
\text { large } \log \dot{E} \\
\text { large } \log B_{\mathrm{LC}} \\
\text { small } Q \\
\text { large } \alpha_{\mathrm{LM}} \\
\text { large } \sin \alpha_{\mathrm{LM}} \\
\text { small } \log B_{\mathrm{LM}}\end{array}$ \\
\hline
\end{tabular}

significant about the pulse emission mechanism and just reflect the strong $P$ dependence in these parameters.

Radio luminosity is anti-correlated with $N F$. Again, an inference of $N F$ correlation with age results since radio luminosity is inversely related to pulsar age (Lyne, Manchester, and Taylor 1985) though we reiterate that there is no explicit correlation between nulling and age. The correlation of both $P$ and $\log \mathcal{L}$ with $N F$ provides indirect evidence for an inverse relationship between $P$ and radio luminosity.

Possibly the most interesting correlation is that between $N F$ and the geometrical parameter $\alpha . \alpha_{L M}$ and $\alpha_{R}$ are highly correlated and so may be discussed together. The correlation suggests that $N F$ increases when $\alpha$ is small. This seems plausible since a radio pulsar may cease to pulse when the magnetic and rotation axes are aligned since there is no fundamental asymmetry in such a system. Lyne and Manchester (1988) find that alignment is a property of older pulsars and again this suggests a correlation of $N F$ with age. However, the situation is not clear since $\alpha_{\mathrm{R}}$ determined by Rankin (1990) does not appear to be related to pulsar age. Also, the model of Beskin et al. (1988) suggests that pulsars counter-align with age and so the correlation may be purely geometrical in origin.

The strong correlation between $N F, B_{\mathrm{LM}}$ and $B_{\mathrm{R}}$ appears to suggest that large surface magnetic fields are associated with increased pulse nulling. This correlation should not be taken at face value because the normalization by $\sin \alpha$ substantially increases $B$ for the pulsars that generally have large $N F$. This also combines with the latent $P$ dependence in $B$ to produce the observed correlation.

For the groups composed of the core emission pulsars, cone emission pulsars, and both types, no significant correlations were found between $p_{\mathrm{s}}$ and the pulsar parameters using standard correlation methods. The expected inverse correlation was found between $p_{\mathrm{s}}$ and $N F$ and had a significance of 0.0194 with Cox's method and 0.0251 with the generalized Kendall's $\tau$.

There is also indirect evidence that the pulse emission mechanism is broadband from $408 \mathrm{MHz}$ to $843 \mathrm{MHz}$ because of the consistency of $N F$ and $p_{\mathrm{s}}$ in the pulsars common to the studies by Biggs (1986) and Ritchings (1976). This is consistent with simultaneous dual frequency observations that have shown significant correlations between subpulses observed over wide frequency ranges (Bartel et al. 1981, and the references therein). However, the situation is more complicated because simultaneous dual frequency observations of PSR 0809+74 have shown that all null pulses observed at $408 \mathrm{MHz}$ are also null when observed at $102 \mathrm{MHz}$, but not vice versa (Davies et al. 1984). 